\title{
Sensitivity analysis of solute kinetics in a four compartmental model for hemodialysis patients
}

\author{
Mohammad Munir ${ }^{1, *}$, Usman Saleem Khan Panni ${ }^{2}$, Nasreen Kausar ${ }^{3}$, Rukhshanda Anjum ${ }^{4}$ \\ 1,2Dept. of Mathematics Govt. Postgraduate College, Abbottabad, Pakistan. \\ ${ }^{3}$ Yildiz Technical University,Faculty of Arts and Science, Department of Mathematics, Esenler, \\ 34210, Istanbul, Turkey. \\ ${ }^{4}$ Dept. of Mathematics and Statistics, University of Lahore, Lahore, Pakistan. \\ *Corresponding author:dr.mohammadmunir@gmail.com
}

\begin{abstract}
Sensitivity Analysis of the most advanced four compartmental mathematical model explaining solute kinetic in the hemodialysis patients was performed on the basis of the data collected from six patients with different Body Mass Indices (BMIs). The toxin concentration in all compartments increases with the decrease in the BMIs of the patients. The clearance rate, $\mathrm{k}_{\text {clear }}$, and the volume of extracellular compartment, $\mathrm{V}_{\mathrm{E}}$, are the most sensitive while the volume of the muscle tissue compartment, $\mathrm{V}_{\mathrm{MT}}$, and the clearance rate, $\mathrm{k}_{\mathrm{MT}}$, are the least sensitive parameters during dialytic interval. The production rate, $G$, and the volume of the extracellular compartment, $\mathrm{V}_{\mathrm{E},}$ are the most sensitive while kclear and $\mathrm{k}_{\mathrm{E}, \mathrm{AT}}$ are the least sensitive parameters of all parameters during the interdialytic interval. The overall production rate, $G$, remains more sensitive than the clearance rate, $\mathrm{k}_{\text {clear }}$ during one complete cycle.
\end{abstract}

Keywords: Body mass index; compartmental model; sensitivity analysis; solute kinetic; toxin concentration.

\section{Introduction}

The kidneys are two bean-shaped organs located on the left and right sides in the retro-peritoneal space in the human. The anatomy of a kidney is shown in Figure (1), (Betts et al., 2013). Their sizes are about 11 centimeters in length. They receive blood from the paired renal arteries, filter it and exit it into the paired renal veins. Each kidney is attached to a ureter that carries excreted urine to the bladder. The kidneys control the volume of various body fluid compartments, fluid osmolality, and acid-base balance, concentrations of various electrolyte and removal of toxins from the blood. The dysfunction or the damage of the kidneys is called the Chronic Kidney Disease (CKD); it means that the kidneys cannot filter blood properly. The main risk factors for developing kidney disease are diabetes, high blood pressure, heart disease and a family history of kidney failure. Filtration occurs in the glomeruli. One-fifth of the blood volume that enters the kidneys is filtered. The kidney's working condition is measured by calculating the estimated Glomerular Filtration Rate (GFR). CKD may come in one of the five stages as described:

Table 1. Description of GFR

\begin{tabular}{|l|l|l|}
\hline Stages & Description & GFR \\
\hline Stage 1 & With normal & $\geq 90 \mathrm{~mL} / \mathrm{min}$ \\
\hline Stage 2 & Mild CKD & $60-89 \mathrm{~mL} / \mathrm{min}$ \\
\hline Stage 3A & Moderate CKD & $45-59 \mathrm{~mL} / \mathrm{min}$ \\
\hline Stage 3B & Moderate CKD & $30-44 \mathrm{~mL} / \mathrm{min}$ \\
\hline Stage 4 & Severe CKD & $15-29 \mathrm{~mL} / \mathrm{min}$ \\
\hline Stage 5 & End Stage CKD & $\leq 14 \mathrm{~mL} / \mathrm{min}$ \\
\hline
\end{tabular}

The kidney is a very complex organ and mathematical modeling has been used to better understand the function of the kidneys at several scales, including fluid uptake and secretion (Weinstein, 1994), (Thomas, 2005). Several mathematical models were presented in the past to understand the Hemodialysis (HD) which removes the toxins from the blood of the patients. The 
mathematical models consist of parameters which describe the intrinsic properties of the kidney system The estimation or identification of these parameters is essential to get full insight into the function of the kidney. Both the parameter estimation and identification require the sensitivity studies of the model output with respect to the parameters present in the model. Various models were presented by different researchers showing the kinetics of the concentration of toxins in the hemodialysis patients while undergoing dialysis.

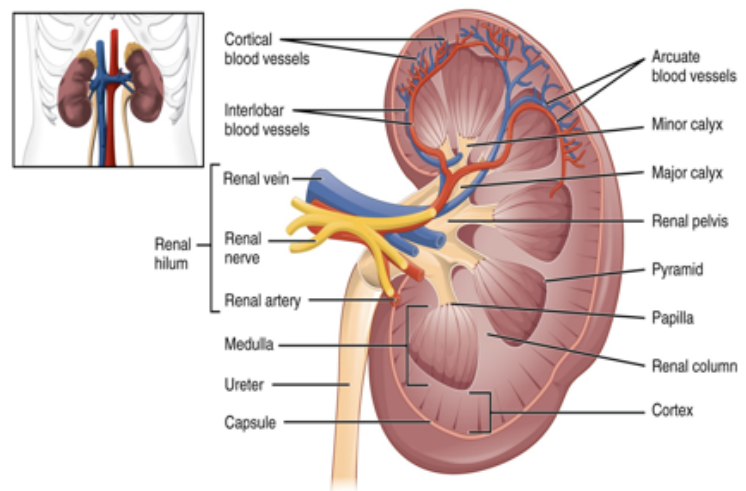

Fig.1. Anatomy of Kidney

The history of mathematical modeling of the hemodialysis is not so old. One of the significant models explaining solute kinetic in hemodialysis patients is the Cronin's model (Cronin-Fine et al., 2007); describing toxin concentrations in three compartments namely the Organ mass compartment (OM), the Muscle Mass and Adipose Tissue compartment (MMAT) and the Extracellular (E) compartment. We analyze the most advanced four-compartmental model given by Kappel et al., (Kappel et al., 2009). Kappel's model is the widely studied Ordinary Differential Equations (ODEs) model. It is four compartmental delineating the muscles and adipose tissue compartments, which were kept in one compartment in the past models. It includes nine important parameters which can be estimated. It simulates the toxin concentration in different compartments for both the dialytic and the interdialytic intervals.

For this purpose, we have divided our article into four sections. In section (1), we describe the hemodialysis and its four-compartmental model along with the concept of sensitivity analysis. In section (2), we explain the methods and parameters used in this article. In section (3), we give our major results. In section (4), we conclude our article.

\subsection{Hemodialysis}

The hemodialysis refers to a process which is done as an alternative of kidney's function (Munir et al., 2018) when the patients have chronic kidney disease (CKD) with Stage 5, i.e. when the Glomerulus Filtration Rate (GFR) of the kidney is less than $15 \mathrm{~mL} / \mathrm{min}$ or the amount of serum creatinine is greater than $6 \mathrm{mg} / \mathrm{dL}$ as mentioned in (Tierney et al., 2016) and (Daugirdas et al., 2007). This process is done on the regular basis to remove waste products such as uremic toxins and excess fluids, also it keeps chemicals such as potassium, sodium, bicarbonate, etc. vin the blood at a safe level. Various models were presented by different researchers showing the kinetics of the concentration of the toxin in the hemodialysis patients while undergoing dialysis. F. Kappel has given the most advanced four compartmental model (Kappel et al., 2009), which was later verified by R. Hussain in (Hussain et al., 2012) using

the data collected from six different patients. The main objectives of this study are to analyze the sensitivities of the parameters involved and to evaluate the most and least sensitive parameters. Data collected from six different patients with different Body Mass Index (BMI) See, (Hussain et al., 2012) were used to solve four compartmental model with the compartments as Organ Mass Compartment (OM), Muscle Tissue Compartment (MT), Adipose Tissue Compartment (AT) and Extracellular Compartment $(E)$, and the sensitivities of all parameters involved in the model have been evaluated for each patient during the dialytic and interdialytic interval, separately.

It is considered that patients on dialysis with high BMI have a greater chance of survival as compared to patients with low 
BMI. But according to (Hussain et al., 2012), BMI is not able to predict the toxin concentration, so body composition is used. Four Compartmental Model (FCM) is used to obtain the results using data from six individual hemodynamically stable patients. It is always of great importance to establish a more accurate model, which can be done by checking all the parameters of the four compartmental model so that the most and the least sensitive parameters, highly influencing the solute kinetic, can be determined.

Patients typically require $H D$ three times a week. Sessions last 3-5 hours depending on patient size, type of dialyzer used, and other factors (Tierney et al., 2016). Here we will consider a treatment of 3.5 hours three times a week. So, the dialytic interval (during dialysis) is 3.5 hours or 210 minutes while the interdialytic interval (during two dialysis) is taken as 2.19 days or 52.5 hours or 3150 minutes (Hussain et al., 2012) and (Kappel et al., 2009).

\subsection{Model}

The four compartmental model is same as given in (Hussain et al., 2012) and (Kappel et al., 2009) which is composed of the following compartments:

\section{Organ Mass Compartment (OM) \\ II. Muscle Tissue Compartment (MT) \\ III. Adipose tissue Compartment (AT) \\ IV. Extracellular Compartment (E)}

Here two assumptions are considered to be true:

a. Dialysis interacts only with $E$ compartment where blood is major part of $E$ compartment so exchange of toxins happens only between compartment $\mathrm{E}$ and compartments $O M, M T$ and $A T$.

b. The exchange of toxins among the compartments occurs according to the principle of concentration gradient (Choy et al., 1974). This principle states that toxins flows from a compartment of high toxin- concentration to a compartment of low toxin-concentration.

The first assumption explains that all compartments other than $E$ compartment exchange the toxins with only $E$ compartment which results in at most three state variables for the first three differential equations governing the rate of toxin concentration in FCM. While the last equation of FCM involves all four state variables since exchange of toxin happens in $E$ compartment and the other three compartments only, according to first assumption. Secondly, exchange of toxins other than the process of diffusion were neglected. Since the concentrations of the solutes before dialysis are comparatively high in the $E$ compartment, as compared to dialysate and constant replacement of the dialysate during dialysis ensures that the concentration of undesired solutes is kept low in the dialysate continuing the removal of toxins from $E$ compartment into dialysate, which allows the removal of toxin in $E$ compartment from the other compartments and eventually from the patient's body. But during interdialytic period, there is no removal of toxin from $E$ compartment so the process is reversed, i.e. the toxin from $E$ compartment diffuse back into the other three compartments where they start accumulating until the dialysis is done. So, the system of four differential equations describing the dynamics of concentration of toxins are given by (Kappel et al., 2009):

$$
\begin{gathered}
\dot{C}_{\mathrm{OM}}=\frac{G}{V_{\mathrm{OM}}}-\frac{k_{\mathrm{E}, \mathrm{OM}}}{V_{\mathrm{OM}}} C_{\mathrm{OM}}+\frac{k_{\mathrm{E}, \mathrm{OM}}}{V_{\mathrm{OM}}} C_{\mathrm{E}}, \\
\dot{C}_{\mathrm{MT}}=-\frac{k_{\mathrm{E}, \mathrm{MT}}}{V_{\mathrm{MT}}} C_{\mathrm{MT}}+\frac{k_{\mathrm{E}, \mathrm{MT}}}{V_{\mathrm{MT}}} C_{\mathrm{E}}, \\
\dot{C}_{\mathrm{AT}}=-\frac{k_{\mathrm{E}, \mathrm{AT}}}{V_{\mathrm{AT}}} C_{\mathrm{AT}}+\frac{k_{\mathrm{E}, \mathrm{AT}}}{V_{\mathrm{AT}}} C_{\mathrm{E}}, \\
\dot{C}_{\mathrm{E}}=\frac{k_{\mathrm{E}, \mathrm{OM}}}{V_{\mathrm{E}}} C_{\mathrm{OM}}+\frac{k_{\mathrm{E}, \mathrm{MT}}}{V_{\mathrm{E}}} C_{\mathrm{MT}}+\frac{k_{\mathrm{E}, \mathrm{AT}}}{V_{\mathrm{E}}} C_{\mathrm{AT}}-(1) \\
\frac{\left(k_{\mathrm{E}, \mathrm{OM}}+k_{\mathrm{E}, \mathrm{MT}}+k_{\mathrm{E}, \mathrm{AT}}+\delta k_{\mathrm{clear}}\right)}{V_{\mathrm{E}}} C_{\mathrm{E}}
\end{gathered}
$$

First equation shows the rate of generation of toxins in $O M$ compartment as well as the rate of exchange of toxins between $O M$ and 
$E$ compartment. The second and third equations shows only the rate of exchange of toxins between $M T$ and $E$ compartments as well as $A T$ and E compartments. Fourth equation shows the rate of exchange of toxins between each compartment and $E$ compartment. It also shows the rate of removal of toxins from $E$ compartment. The constant $\delta=1$ for dialytic intervals and $\delta=$ 0 for the inter-dialysis intervals. The detail and description of all the parameters and state variables used in the model (1) is given in the following Table 2.The solution of the

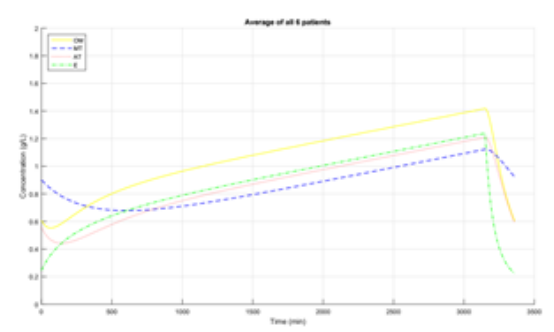

Fig. 2. Solution of the Model for Parametric Value of Average Patient

Table 2. Nomenclature of the variables and parameters used in the model

\begin{tabular}{|c|c|c|}
\hline Parameter & Description & Unit \\
\hline BW & Body Weight & $\mathrm{Kg}$ \\
\hline BMI & Body Mass Index & $\mathrm{Kg} / \mathrm{m}^{2}$ \\
\hline$G$ & Toxin Production Rate & $\mathrm{gm} / \mathrm{min}$ \\
\hline$V_{O M}$ & $\begin{array}{l}\text { Volume of Organ Mass } \\
\text { Compartment }\end{array}$ & $\mathrm{L}$ \\
\hline$V_{M T}$ & $\begin{array}{l}\text { Volume of Muscle } \\
\text { Tissue Compartment }\end{array}$ & $\mathrm{L}$ \\
\hline$V_{A T}$ & $\begin{array}{l}\text { Volume of Adipose } \\
\text { Tissue Compartment }\end{array}$ & $\mathrm{L}$ \\
\hline$V_{E}$ & $\begin{array}{l}\text { Volume of Extracellular } \\
\text { Compartment }\end{array}$ & $\mathrm{L}$ \\
\hline$C_{O M}$ & $\begin{array}{l}\text { Concentration of Toxins } \\
\text { in OM Compartment }\end{array}$ & $\mathrm{gm} / \mathrm{L}$ \\
\hline$C_{M T}$ & $\begin{array}{l}\text { Concentration of Toxins } \\
\text { in MT Compartment }\end{array}$ & $\mathrm{gm} / \mathrm{L}$ \\
\hline$C_{A T}$ & $\begin{array}{l}\text { Concentration of Toxins } \\
\text { in AT Compartment }\end{array}$ & $\mathrm{gm} / \mathrm{L}$ \\
\hline$C_{E}$ & $\begin{array}{l}\text { Concentration of Toxins } \\
\text { in E Compartment }\end{array}$ & $\mathrm{gm} / \mathrm{L}$ \\
\hline$k_{E, O M}$ & $\begin{array}{l}\text { Rate Constant of } \mathrm{E} \text { and } \\
\text { OM Compartments }\end{array}$ & $\mathrm{L} / \mathrm{min}$ \\
\hline$k_{E, M T}$ & $\begin{array}{l}\text { Rate Constant of E and } \\
\text { MT Compartments }\end{array}$ & $\mathrm{L} / \mathrm{min}$ \\
\hline$k_{E, A T}$ & $\begin{array}{l}\text { Rate Constant of E and } \\
\text { AT Compartments }\end{array}$ & $\mathrm{L} / \mathrm{min}$ \\
\hline$k_{\text {clear }}$ & Dialysis Clearance Rate & $\mathrm{L} / \mathrm{min}$ \\
\hline
\end{tabular}

model is shown in Figure (2) for a full one cycle.

\subsection{Sensitivity Analysis}

Sensitivity analysis is the evaluation of the sensitivities of the model output with respect to the parameters of the model. The sensitivity function of a model output with regard to a parameter describes the effects of the changes in the value of the parameter on the value of the output of the model. For example: it can be used to know how the dose level of a drug changes with respect to changes in the values of the parameter like toxity rate (Koc et al., 2020). Goyal et al., (Goyal et al., 2018) used it to assess the quality of air and refrigeration. Basically, sensitivity is a unit less ratio of the relative error in function to the relative error in the parameter.

The consistency between the results given by sensitivity analysis and other analyses build up the findings and conclusions of a model. Sensitivity analysis quantifies how much the outcomes of the models are robust to the changes in the inputs (Thabane et al., 2013).

A single output of the model is generally given by the equation see (Batzel et al., 2007), (Munir, 2019)):

$y_{j}(t)=f_{j}(t, \theta), \quad 0 \leq t \leq T, j=1, \ldots, m$.

$\theta=\left(\theta_{1}, \ldots, \theta_{p}\right)^{T}$ is a vector of model parameters and $f_{j}, j=1, \ldots, m$, is a sufficiently smooth function. We want to know the changes in the value of the function $f_{j}(t, \theta), j$ $=1, \ldots, m$, with respect to a single parameter $\theta_{i}, i=1, \ldots, p$. For this purpose, we define the sensitivity of the given model output $f_{j}(t$, $\theta$ ) with respect to a given parameter $\theta_{i}, i=$ $1, \ldots p$ denoted by $S f_{j} / \theta_{i}$, (Munir, 2017), as:

$$
S_{f_{j} / \theta_{i}}=\lim _{\Delta \theta \rightarrow 0} \frac{\frac{f_{j}\left(t, \theta_{i}+\Delta \theta_{i}\right)-f_{j}\left(t, \theta_{i}\right)}{f_{j}\left(t, \theta_{i}\right)}}{\frac{\left(\theta_{i}+\Delta \theta_{i}-\theta_{i}\right)}{\theta_{i}}},
$$




$$
\begin{gathered}
S_{f_{j} / \theta_{i}}=\lim _{\Delta \theta \rightarrow 0} \frac{\frac{\Delta f_{j}}{f_{j}}}{\frac{\Delta \theta_{i}}{\theta_{i}}}, \\
S_{f_{j} / \theta_{i}}=\lim _{\Delta \theta \rightarrow 0} \frac{\Delta f_{j}}{\Delta \theta_{i}} \frac{\theta_{i}}{f_{j}}, \\
S_{f_{j} / \theta_{i}}=\frac{\partial f_{j}}{\partial \theta_{i}} \frac{\theta_{i}}{f_{j}}, j=1, \ldots, m, \quad i=1, \ldots, p
\end{gathered}
$$

where $f_{j}, j=1, \ldots, \mathrm{m}$ is a single model output, $\theta_{i}, i=1, \ldots, p$ a single model parameter, $f_{j}$ and $\theta_{i}$ are the values around which changes are computed. Note that in this formulation time is fixed so that sensitivities are functions of time over the time interval of interest. Also, note that these quantities provide the local estimates of sensitivity. The global behavior of the model output due the changes in the parameter is usually given by the system sensitivity.

Definition 1.4 The system sensitivity with respect to a single parameter at any time instant $t$ in $[0, T]$ taking together the separate sensitivities of all model outputs $f_{j}, j=1, \ldots$, $m$ is defined by, (See Ref: (Munir, 2018)):

$$
S_{\theta_{i}}=\left(\sum_{j=1}^{m}\left(S_{\frac{f_{j}}{\theta_{i}}}(t)\right)^{2}\right)^{\frac{1}{2}}, i=1, \ldots, p
$$

\section{Simulations}

In this section, first we will discuss the methods and techniques for plotting sensitivity graphs as well as solution graphs. Also, we will discuss the parameter values used for simulations.

\subsection{Method}

Data of six different hemodynamically stable patients are taken from (Hussain et $a l ., 2012$ ) and is used to calculate and plot the solution and sensitivity graphs using $M A T L A B$ Ordinary Differential Equations $(O D E)$ solver, ode45. The ode45 uses Runge
Kutta of order 4 and 5 to find the numerical solution of the Initial Value Problems (IVPs), which in this paper are the four simultaneous linear differential equations given by (1) referring to one compartment each. However, one of the three methods discussed in (Majeed, 2020) can be used. While the partial differentiation of equations required for sensitivity are done manually whereas ode45 is used for plotting their graphs. The sensitivity graphs are separately plotted for both interdialytic and dialytic intervals respectively. The solution graphs are plotted collectively for one complete cycle including one interdialytic and one dialytic interval, given in Figure (2), which are similar to the graphs as given in the literature (Hussain et al., 2012). Here 'Direct Method' is used to estimate the sensitivity coefficients. The direct method is based on considering all parameters as constants and then the sensitivity coefficients are estimated by solving sensitivity equations simultaneously with the original system. If all the parameters appearing in the system model given by the set of Equation (1) are taken to be constants, then sensitivity analysis, in this case, may just entail finding the partial derivatives of the solution with respect to each parameter. Which in this case are the partial derivatives of state variables $\left(C_{O M}, C_{M T}, C_{A T}\right.$ and $C_{E}$ ) with respect to the parameters $V_{O M}, V_{M T}$, $V_{A T}, V_{E}, k_{E}, O_{M}, k_{E}, M T, k_{E}, A T, k_{\text {clear }}$ and $G$. In order to find the sensitivities of these four state variables with respect to these nine parameters over the time interval for one cycle as $(0 \leq t \leq 3360)$ for the periodic solution, we proceed as per our procedure given in Subsection 1.3 to arrive at Equation (3) which specifies the sensitivity of a state variable with respect to a parameter. As an illustration, we consider $\theta_{i}=V_{O M}, f_{1}(t, \theta)$ $=C_{O M}, f_{2}(t, \theta)=C_{M T}, f_{3}(t, \theta)=C_{A T}$ and $f 4(t, \theta)=C E$, then the partial derivatives of the solution $C_{O M}, C_{M T}, C_{A T}$ and $C_{E}$ with respect to $V_{O M}$ give rise to following set of sensitivity equations, (Kappel \& Munir, 2017): 


$$
\begin{gathered}
\dot{S}_{1}=\left(-k_{\mathrm{E}, \mathrm{OM}} S_{1} V_{\mathrm{OM}}+k_{\mathrm{E}, \mathrm{OM}} C_{\mathrm{OM}}+\right. \\
\left.k_{\mathrm{E}, \mathrm{OM}} S_{4} V_{\mathrm{OM}}+k_{\mathrm{E}, \mathrm{OM}} C_{\mathrm{E}}-G\right) /\left(V_{\mathrm{OM}}\right)^{2} \\
\dot{S}_{2}=\left(k_{\mathrm{E}, \mathrm{MT}}\left(S_{4}-S_{2}\right)\right) / V_{\mathrm{MT}} \\
\dot{S}_{3}=\left(k_{\mathrm{E}, \mathrm{AT}}\left(S_{4}-S_{3}\right)\right) / V_{\mathrm{AT}} \\
\dot{S}_{4}=\left(k_{\mathrm{E}, \mathrm{OM}} S_{1}+k_{\mathrm{E}, \mathrm{MT}} S_{2}+k_{\mathrm{E}, \mathrm{AT}} S_{3}-\right. \\
\left.\left(k_{\mathrm{E}, \mathrm{OM}}+k_{\mathrm{E}, \mathrm{MT}}+k_{\mathrm{E}, \mathrm{AT}}+\delta k_{\mathrm{clear}}\right) S_{4}\right) / V_{\mathrm{E}}
\end{gathered}
$$

where $S_{1}=\partial C_{O M} \partial V_{O M}, S_{2}=\partial C_{M T} / \partial V_{O M}, S_{3}=$ $\partial C_{A T} / \partial V_{O M}, S_{4}=\partial C_{E} / \partial V_{O M}$.

Then, this system of sensitivity Equations (5) along with the original system of Equations (1) is solved to estimate the sensitivity of the state variables $\left(C_{O M}, C_{M T}, C_{A T}, C_{E}\right)$ with respect to the parameter $V_{O M}$. The similar procedure and argument holds for estimating the sensitivity of the state variables with respect to the parameters $V_{O M}, V_{M T}, V_{A T}$, $V_{E}, k_{E, O M}, k_{E}, M T, k_{E, A T}, k_{c l e a r}$ and $G$, which results in 36 simultaneous equations. So, these simultaneous equations are solved along with the original system of Equations (1) using ode45 and then each 4 equations are plotted resulting in nine graphs, one graph for each parameter.

\subsection{Parameters}

The volumes of all four compartments are shown in the table in decreasing order with

\begin{tabular}{|c|c|c|c|c|c|c|c|c|}
\hline है & VoM & $V_{M} T$ & $V_{A T}$ & $V_{E}$ & $G$ & $k_{\text {clear }}$ & BW & BMI \\
\hline 1 & 4.4 & 23.175 & 4.31 & 28.5 & 0.0128 & 0.276 & 108 & 42.3 \\
\hline 2 & 4.9 & 20.775 & 4.36 & 18.4 & 0.0108 & 0.298 & 100 & 36.2 \\
\hline 3 & 1.8 & 22.95 & 2.78 & 21.6 & 0.0088 & 0.320 & 93 & 29.8 \\
\hline 4 & 4.5 & 18.375 & 2.24 & 17.8 & 0.0061 & 78 & 26.0 & 26.0 \\
\hline 5 & 3.4 & 13.125 & 1.27 & 16.2 & 0.0072 & 58 & 21.3 & 21.3 \\
\hline 6 & 3.0 & 12 & 1.48 & 14.8 & 0.0081 & 54 & 20.5 & 20.5 \\
\hline
\end{tabular}
respect to body weights and BMI. The table also shows the individual clearance and production rate of all six patients.

Table 3. Different Parameter Values for the patients enrolled in the study

Table 4. Average Initial Values and Rate Constants

\begin{tabular}{|c|c|c|c|c|c|c|}
\hline COM & COM & COM & COM & $k E, O M$ & $k E, M T$ & $k E_{, A T}$ \\
\hline 0.61 & 0.91 & 0.56 & 0.25 & 0.046 & 0.032 & 0.019 \\
\hline
\end{tabular}

\section{Results}

\subsection{Interdialytic Interval}

For parameters $V_{O M}, V_{M T}, V_{A T}$ and $V_{E}$, all the four compartments show increased sensitivities with decrease in BMI during interdialytic interval. For parameter $k_{E}, O M$, only I shows an increased behavior while the $C_{O M}$ and $C_{E}$ concentrations show a decreasing trend whereas the $C_{A T}$ remains insensitive with decreasing BMI. All concentrations show an increasing trend for $k_{E}, M T$ parameter during interdialytic interval. All the concentrations in the four compartments show a decrease in their sensitivities except $C_{M T}$ which remains insensitive with the decrease in BMI for $k_{E, A T}$ parameter. For the parameter kclear, the sensitivities of all the concentrations remain constant or unchanged with the decrease in the BMI during interdialytic interval. The sensitivities of all the concentrations increase as the BMI decreases for the parameter I during interdialytic interval.

For average data during interdialytic interval as shown in the Figure (3), the $C_{O M}$ is the most sensitive for parameters $V_{O M}, k_{E, O M}$ and $G$. The concentration $C_{M T}$ is most sensitive for parameters $V_{M T}$ and $k_{E, M T}$. Similarly, the $C_{A T}$ is the most sensitive for parameters $V_{A T}$ and $k_{E, A T}$. The remaining concentration $E$ is the most sensitive for only $V_{E}$ parameter.

System sensitivities for average data, as discussed before, during interdialytic interval is shown in Figure (5), which shows the parameters $G, V_{E}$ and $k_{E, O M}$ are the most sensitive of all the parameters while the least sensitive parameters are kclear, $k_{E, A T}$ and $V_{A T}$.

\subsection{Dialytic Interval}

The parameter VOM shows a trend of increasing sensitivity for all compartments with decreasing BMI during dialytic interval. The parameter $V_{M T}$, shows similar results 
with the decrease in BMI during dialytic interval. The sensitivities of the concentrations $C_{O M}, C_{A T}$ and $C_{E}$ increase for the parameter $V_{A T}$ while the remaining concentration $C_{M T}$ remains constant as BMI changes. All the concentrations show increase in their sensitivities except $C_{O M}$ with decreasing BMI for parameter $V_{E}$ during dialytic interval. The $C_{M T}$ and $C_{A T}$ concentrations remain insensitive while other two concentrations show increased sensitivities with the decrease in BMI, for parameter $k_{E, O M}$. The parameter $k_{E, M T}$, shows a trend of increasing sensitivity for all concentrations with the decrease in BMI during dialytic interval. All concentrations except $C_{M T}$ show an increasing trend with respect to decrease in BMI for the parameter $k_{E, A T}$. For parameter kclear, the sensitivity of all concentrations shows an increasing trend in their sensitivities with decrease in BMI. Similarly, all the concentrations show increasing sensitivities with decrease in BMI for the parameter $G$.
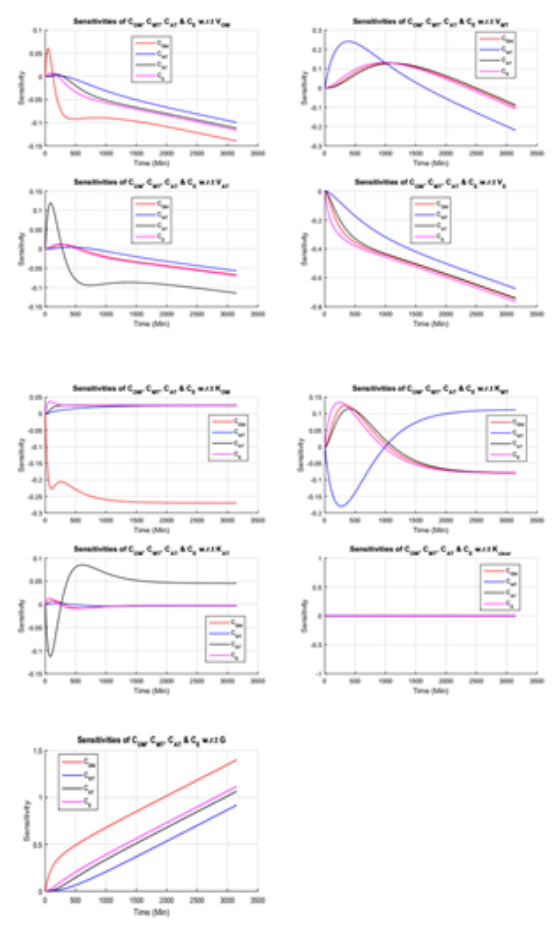

Fig.3. Interdialytic Interval for Average Patient
For average data during dialytic interval as shown in the Figure (6), $C_{O M}$ is the most sensitive for parameters $V_{O M}, k_{E, O M}$ and $G$. The $C_{M T}$ is the most sensitive for parameters $V_{M T}$ and $k_{E, M T}$. Similarly, the $C_{A T}$ compartment is the most sensitive for parameters $V_{A T}$ and $k_{E, A T}$. The remaining concentration $C_{E}$ is the most sensitive for parameters $V_{E}$ and $k_{\text {clear }}$.

System sensitivities as evaluated by using Equation (4) for the average data given in Table 4 during dialytic interval is shown in Figure (8), which shows the parameters $I$ are the most sensitive of all the parameters and $V_{M T}, k_{E, M T}$ and $V_{A T}$ are the least sensitive parameters.

\subsection{Sensitivities of Individual Patients}

Since the primary aim of this work was to analyze Kappel's model for sensitivity studies, and to know of the sensitive and insensitive parameters,

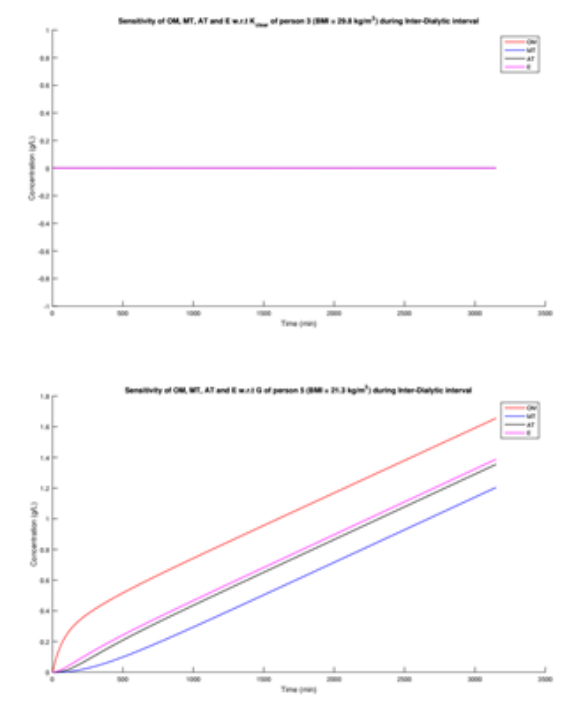

Fig. 4. Interdialytic Interval for Patient-3 and 5

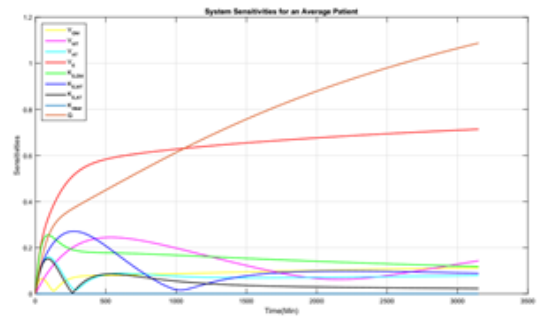

Fig.5. System Sensitivities for an Average Patient during Interdialytic Interval 
we used the average of values of the parameters of the six ambulatory patients on hemodialysis. This helped to study the behavior of toxin concentrations in the hemodialysis patients. Along these lines, we also performed the sensitivity analysis of each patient. The results obtained from the individual sensitivities of the patients were similar, with not a single one deviating from the results of average patients (no outliers). This fact is quite clear from the selected sensitivities of the two arbitrary selected patient Patient-1 and Patient-2; their sensitivities for the selected parameters in the interdialytic and dialytic intervals are shown in Figure (4) and Figure (7) respectively.

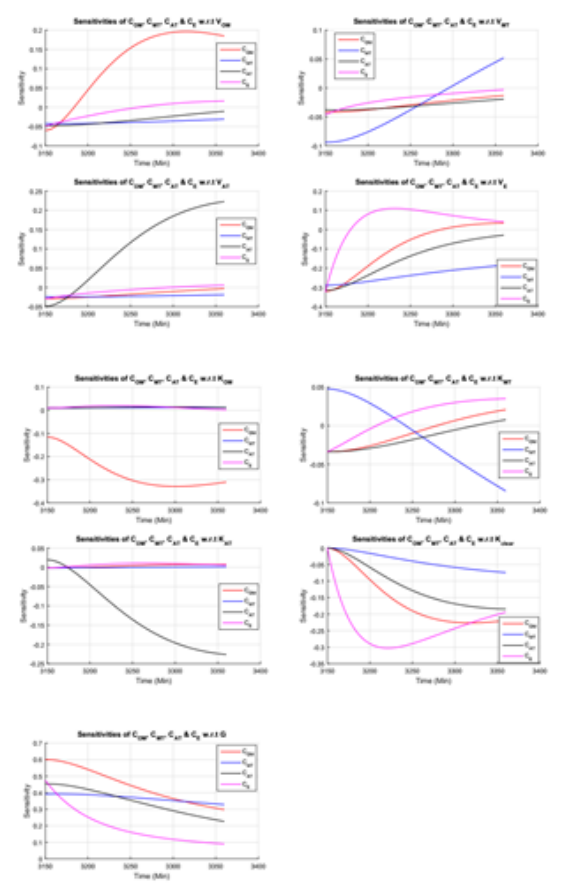

Fig. 6. Dialytic Interval for Average Patient

\section{Conclusions}

The sensitivity of the four concentrations in the four compartmental model describing the dynamics of the toxin for each compartment have been performed. The sensitivities of the parameters for the dialytic and interdialytic interval were evaluated. The main points are as under: i. $\quad$ For both dialytic and interdialytic intervals the sensitivity of $C_{E}$ remains the most prominent one since it occurs as the highest or second highest in each figure. ii. It can also be seen that the sensitivity of almost each concentration increases with the decrease in BMI of patient.

iii. From above two statements we can guess that the slim patients has increased morality rate since slim patients
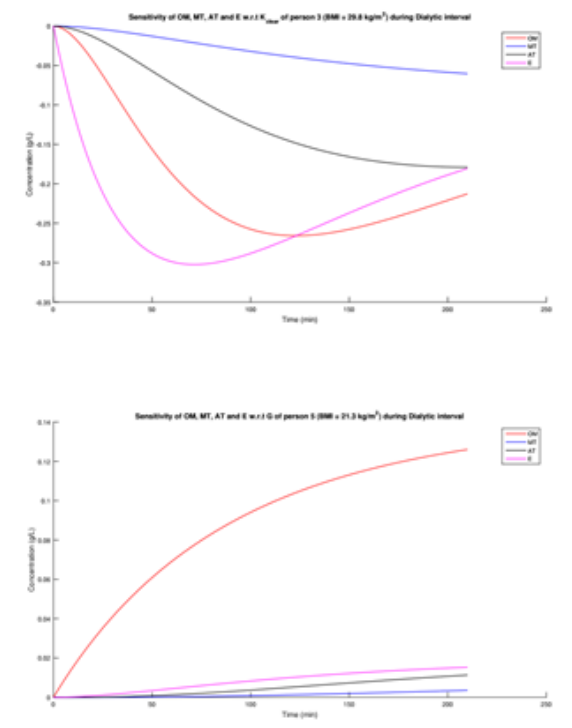

Fig. 7. Dialytic Interval for Patient-3 and 5

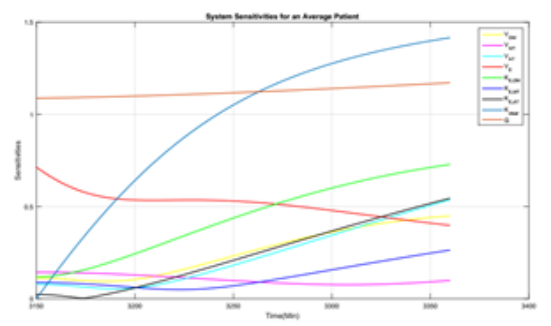

Fig. 8. System Sensitivities for an Average Patient during Dialytic Interval generally have greater volume of $\mathrm{E}$ compartment w.r.t their bodies as compared to obese patients. This effect is, maybe, due to the fact that the patients with higher level of BMI, not only, have more fat mass, but also have more muscle mass. A detailed study on this effect is given in (Abramowitz et al., 2016).

iv. Of all parameters $k_{\text {clear }}$ and $G$ are the most sensitive while $V_{M T}$ and $k_{E, M \mathrm{~T}}$ 
are the least sensitive parameters during dialytic interval.

v. Similarly, $G$ and $V_{E}$ are the most sensitive while $k_{\text {clear }}$ and $k_{E, A T}$ are the least sensitive parameters of all parameters during interdialytic interval.

vi. Clearance rate kclear seems to be more influential than the production rate I during the dialytic interval while during the interdialytic interval the production rate $G$ is more sensitive than clearance rate and clearance rate remains insensitive throughout the interval.

vii. During one complete cycle, the overall production rate $G$ remains more influential than the clearance rate $k_{\text {clear }}$ as is evident from its system sensitivities for dialytic and interdialytic intervals respectively given in figures (8) and (5).

\section{References}

Abramowitz, M. K., Sharma, D., and Folkert, V. W., (2016). Hidden obesity in dialysis patients: clinical implications, in: Seminars in dialysis, Vol. 29, Wiley Online Library, pp. 391-395.

Batzel, J.J., Kappel, F., Schneditz, D. and Tran, H.T., (2007). Cardiovascular and respiratory systems: modeling, analysis, and control (Vol. 34). SIAM.

Betts, J.G., Desaix, P., Johnson, J.E., Korol, O., Kruse, D., Poe, B., Wise, J., Womble, M.D., and Young, K.A., (2013). OpenStax College, et al. Anatomy and Physiology.

Choy, E.M., Evans, D.F., and Cussler, E.L., (1974). Selective membrane for transporting sodium ion against its concentration gradient. Journal of the American Chemical Society, 96(22), pp.7085-7090.

Cronin-Fine, D., Gotch, F., Levin, N.W., Kotanko, P., and Lysaght, M., (2007). A mathematical model comparing solute kinetics in low- and high-BMI hemodialysis patients. The In- ternational journal of artificial organs, 30(11), pp.1000-1007.
Daugirdas, J.T., Blake, P.G., and Ing, T.S. eds., (2007). Handbook of dialysis (Vol. 236). Lippincott Williams \& Wilkins.

Goyal, N., Ram, M., and Banga, S., (2018). Sensitivity assessment of air and refrigeration. Kuwait Journal of Science, 45(1).

Hussain, R., Kappel, F., Zhu, F., Levin, N.W., and Kotanko, P., (2012). Body composition and solute kinetics in hemodialysis patients: a mathematical model. IET Communications, 6(18), pp.3301-3308.

Kappel, F., Batzel, J.J., Bachar, M., and Kotanko, P., (2009). A mathematical model comparing solute kinetics in low and high BMI hemodialysis patients. Institute for Mathematics and Scientific Computing, University of Graz, Tech. Report, (1).

Kappel, F., and Munir, M., (2017). Generalized sensitivity functions for multiple output systems. Journal of Inverse and Ill-posed Problems, 25(4), pp.499-519.

Koc, T., and Cengiz, M.A., (2020). Investigating Different Priors in Bayesian Continual Reassessment Method. Kuwait Journal of Science, 47(1), pp.22-32.

Majeed, A.J., (2020). Three iterative methods for solving Jeffery-Hamel flow problem. Kuwait Journal of Science, 47(1), pp.1-13.

Munir, M., (2017). Sensitivity and Generalized Sensitivity Studies of the SIR and SEIR Models of Computer Virus. Proceedings of the Pakistan Academy of Sciences: A. Physical and Computational Sciences, 54(2), pp.167- 178.

Munir, M., Ali, A., and Hussain, R., (2018). An Improved Mathematical Model of Solute Kinetic During Hemodialysis. Punjab University Journal of Mathematics, 50(1), pp.55-66. 
Munir, M., (2018). Generalized sensitivity analysis of the minimal model of the intravenous glucose tolerance test. Mathematical biosciences, 300, pp.14-26.

Munir, M., (2019). On the Concept of OffDiagonal Generalized Sensitivity Functions and Their Relations to the Parameter Estimates and Correlation. Punjab University Journal of Mathematics, 51(1), pp.61-77.

Thabane, L., Mbuagbaw, L., Zhang, S., Samaan, Z., Marcucci, M., Ye, C., Thabane, M., Giangregorio, L., Dennis, B., Kosa, D., and Debono, V.B., (2013). A tutorial on sensitivity analyses in clinical trials: the what, why, when and how. BMC medical research methodology, 13(1), p.92.

Thomas, S. R., (2005). Modelling and simulation of the kidney, Journal of Biological Physics and Chemistry, 5(2/3), pp.1-14.

Tierney, L.M., McPhee, S.J., and Papadakis, M.A., (2001). Current Medical Diagnosis \& Treatment: Pocket Guide. McGraw-Hill.

Weinstein, A.M., (1994). Mathematical models of tubular transport. Annual review of physiology, 56(1), pp.691-709.

$\begin{array}{lr}\text { Submitted: } & 27 / 01 / 2020 \\ \text { Revised: } & 02 / 05 / 2020 \\ \text { Accepted: } & 16 / 08 / 2020 \\ \text { DOI: } & 10.48129 / \text { kjs.v48i3.9085 }\end{array}$

